\title{
Angiosarcoma of the mandible: Metastasis from a primary tumor of the right atrium of the heart
}

\author{
Anwar Ramadhan, Helena Willén, Andreas Thor ${ }^{*}$ \\ Department of Plastic \& Oral and Maxillofacial Surgery Institute of Surgical Sciences, Uppsala University, Uppsala, Sweden; \\ *Corresponding Author: andreas.thor@akademiska.se
}

Received 27 January 2013; revised 28 February 2013; accepted 8 March 2013

\begin{abstract}
Angiosarcoma of the heart is a rare tumor with very poor prognosis especially in patients with multiple metastases. We present a case of a 39year-old woman with angiosarcoma of the heart metastasing to the right side of the mandible, a situation not often found previously in the literature.
\end{abstract}

Keywords: Angiosarcoma; Heart; Pathological Fracture; Metastasis; Mandible

\section{INTRODUCTION}

Angiosarcoma is a rare malignant tumor of endothelial cells of vascular or lymphatic origin, and characterized as an aggressive tumor with poor prognosis representing less than $1 \%$ in all patients with malignant tumor [1-3]. The angiosarcomas in the oral cavity and salivary glands represent $1 \%$ of all angiosarcomas reported in the literature and are therefore considered as extremely rare [4]. The tumor type is represented evenly between sexes, can occur in all age groups and is overrepresented among older white men with cutaneous lesions [2]. In this paper we report a case of angiosarcoma found in the mandible as a result of metastasis from the primary tumor in the right atrium of the heart.

\section{CASE REPORT}

A 39-year-old white woman was referred to the Department of Oral and Maxillofacial Surgery, Uppsala University Hospital, Uppsala, Sweden, for alteration of sensibility in the right mental nerve and pain from the right side of the mandible. The medical history revealed a primary diagnosis of angiosarcoma of the heart, with metastases to the lung and liver that was established the year before. The angiosarcoma of the heart had surgically been treated with resection of the right atrium the year before submission to our department. Chemotherapy treatment of six different modalities followed as well as radiotherapy for palliation to the lung as well as later to the mandible. The metastasis of the oral cavity was diagnosed after a biopsy following complaints from the patient of pain from the right mandible. Radiation aiming at the affected area of the mandible was commenced.

\section{CLINICAL COURSE AND FINDINGS}

At the time of admittance the patient had fever for a few days. On clinical examination, the patient presented hypoesthesia of the right mental region as well as negative sensibility of the premolars and first molar in the right lower jaw. Soft tissues surrounding these teeth were in normal condition without any signs of swelling. Radiological examination on admission showed a peri-apical radiolucency of the second premolar of the right side of the mandibleand endodontic treatment of the nonsensible teeth was continued (Figure 1). The diagnosis of a metastasis from the angiosarcoma was histologically verified by a biopsy.

The symptoms remained and increased. PET/CT and MRI were performed. The patient reported a swelling buccal to the first molar in the affected region, which disappeared with the initiation of chemotherapy and simultaneous corticosteroid treatment. A second episode of

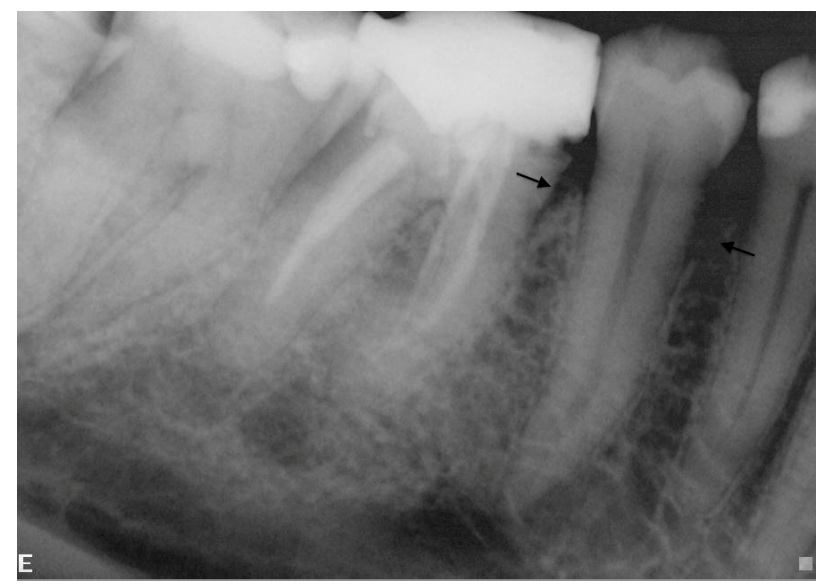

Figure 1. Peri-apical radiograph showing mild resorption of the marginal bone between the premolars and first molar. 
chemotherapy and radiation aimed at the affected region of the mandible was thereafter initiated. During this time the patient had a serious episode of bleeding that required surgery from the area of the metastasis where the biopsy was previously performed. The condition was surgically treated with local hemostasis including fibrin glue, sutures and compression. Radiation and chemotherapy was in summary successful in reducing the tumor, but as a result exposure of bone was thereafter continuously more evident in the buccal area between the premolars in the affected region.

Almost exactly one year after the first contact with our department, the patient developed a pathological fracture at right side of affected area of the mandible (Figure 2). She was admitted for treatment including resection of the affected area and reconstruction surgery with a reconstruction plate (Figure 3). The resected specimen at this time displayed metastasis tumorous tissue as earlier the biopsy showed prior to radiation. General palliation and no further treatment of the oral situation except support from hygienist were thereafter performed. The patient died after surviving 26 months from the first diagnosis of the primary tumor of the heart.

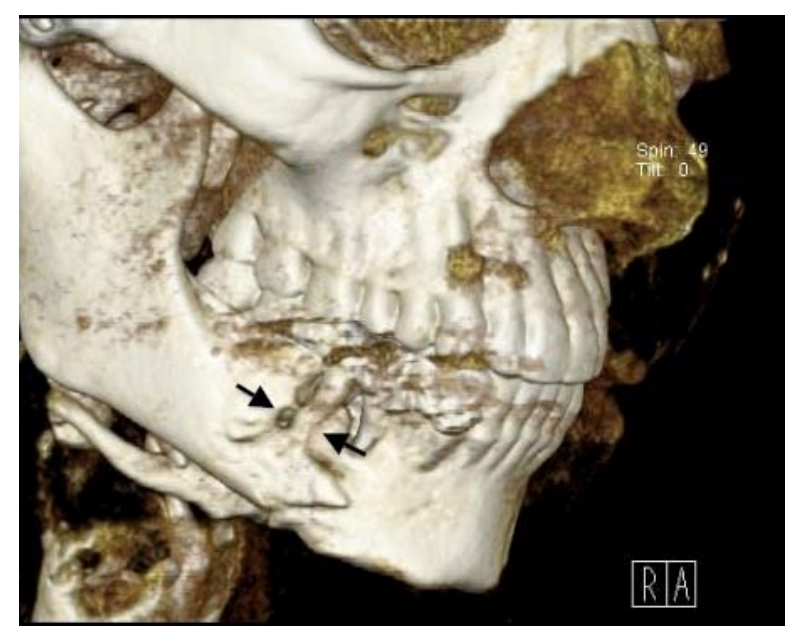

Figure 2. Three-dimensional computed tomography showing pathological fracture located at the right side of the mandible between the first molar and second premolar.

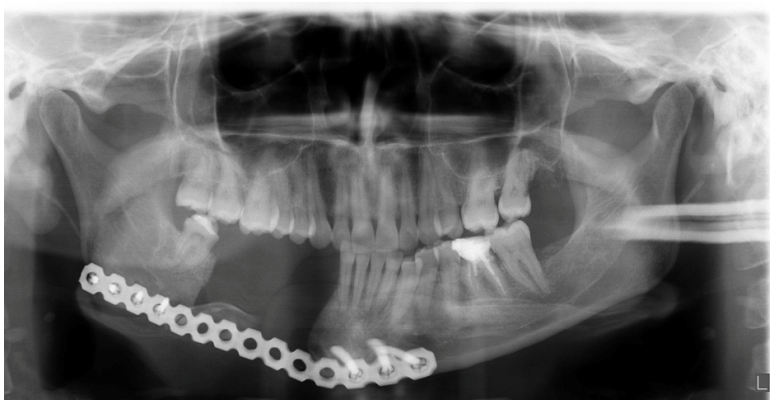

Figure 3. Postoperative oral panoramic radiography showing the resected area and reconstruction plate in place.

\section{PATHOLOGICAL FINDINGS}

\subsection{Macroscopic}

The primary tumor from the right atrium of the heart was a large mass measuring $60 \times 50 \times 40 \mathrm{~mm}$ with a weight of 54 gram. Macroscopically, it was polypoid mass, hemorrhagic and attached to the underlying atrium tissue with a clot-like mass.

\subsection{Histopathologic Findings}

The tumor had a vasoformative feature with primitive vascular spaces of variable sizes (Figure 4) filled with erythrocytes and/or fibrin. In other areas, the dominating structure was solid. The tumor was composed of cell-rich tissue with a spindle formed sarcomatous cell-population with a high grade of cellular, nuclear and nucleolaratypia and atypical mitotic activity (Figure 5). The surgical margins were not clear and there was both pericardial

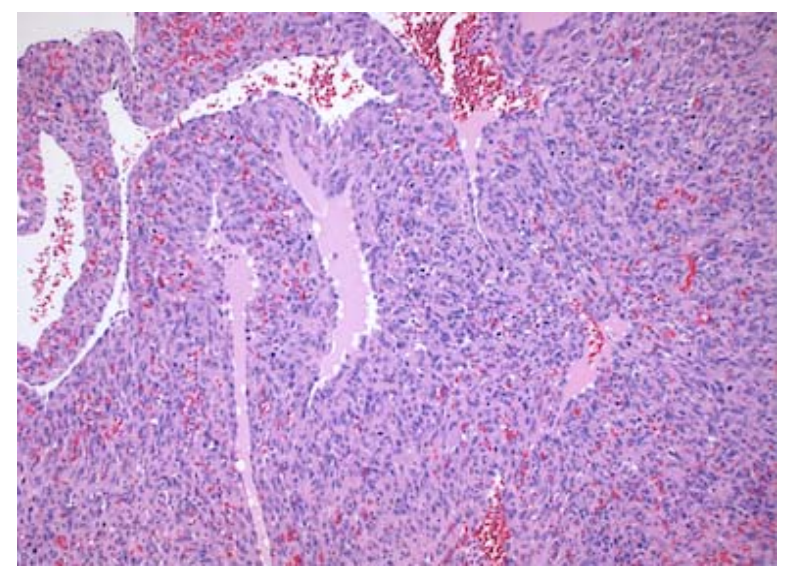

Figure 4. Histological section of the primary angiosarcomaof the right atrium of the heart displaying a solid structure and primitive vascular spaces. Hematoxylin and Eosin. Magnification $\times 100$.

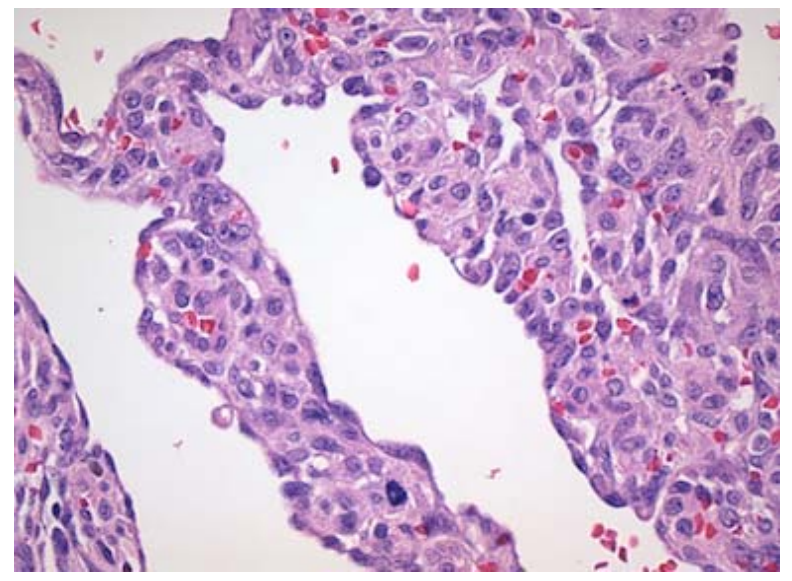

Figure 5. Atypical spindle cell population with atypical mitotic figures in primary tumor of the heart.Hematoxylin and Eosin. Magnification $\times 400$. 
and myocardial tumor infiltration.

\subsection{Immunohistochemistry}

The tumor cells were strongly positive for endothelial cell markers CD31 and CD34 and also for Vimentin. Proliferative activity in Ki-67 staining was very high (Figure 6).

The biopsy from the right mandible showed a metastatic tumor with a similar vasoformative structure (Figure 7), with primitive vascular spaces, spindle cell population with high mitotic activity and strongly positive staining for CD31 (Figure 8).

\section{DISCUSSION}

Primary oral angiosarcomas are often misdiagnosed as other lesions because of their rarity [5].

Accordingly, Munoz et al., who reported a case where

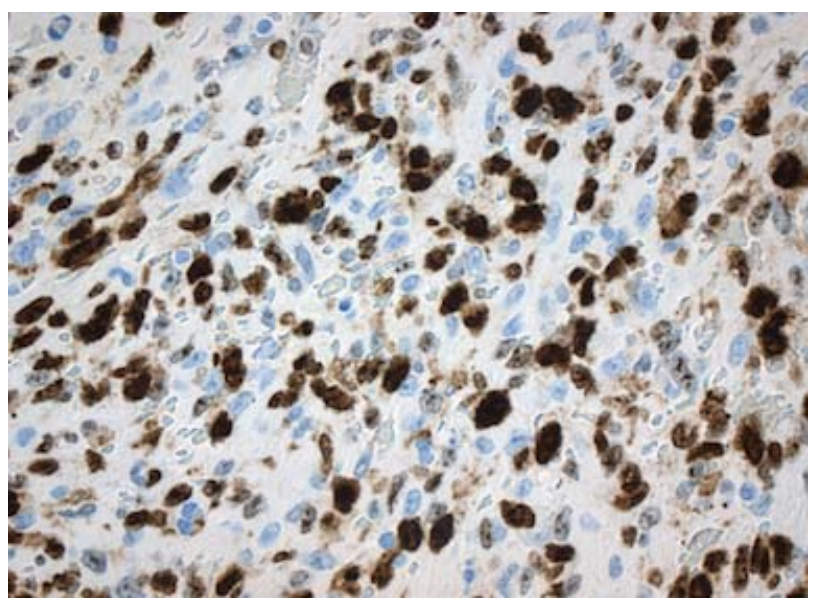

Figure 6. High proliferative activity in Ki-67 staining of the primary angiosarcoma tumor of the right atrium of the heart. Magnification $\times 400$.

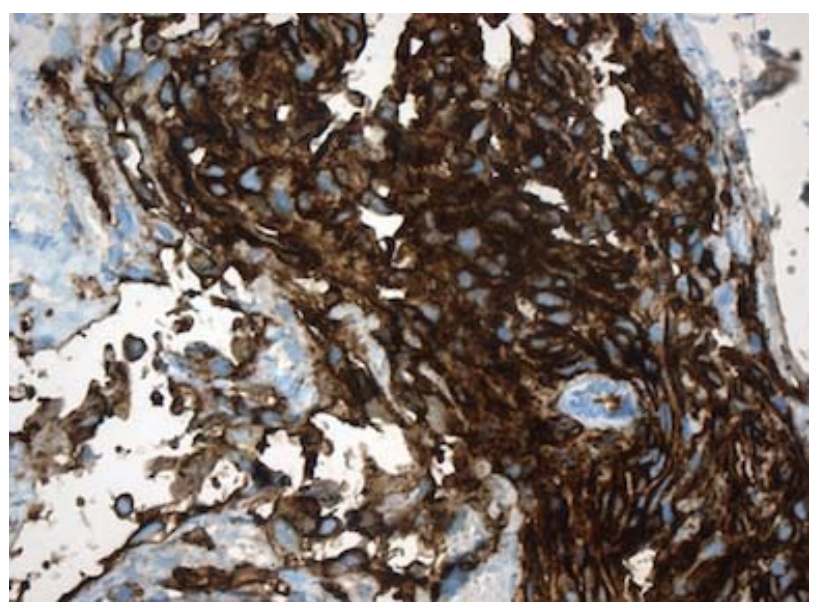

Figure 7. Metastatic tumor in the right mandible with high grade spindle cell atypia and primitive vascular formation. Van Gieson staining. Magnification $\times 400$.

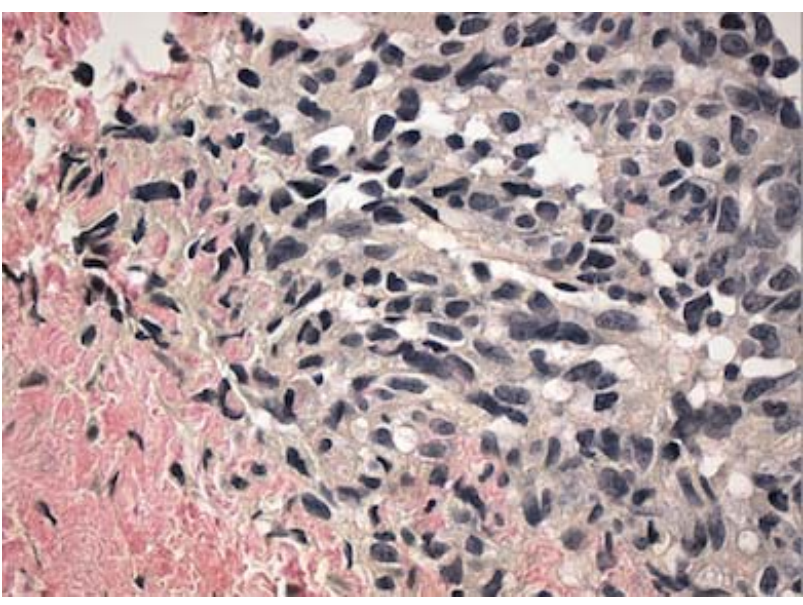

Figure 8. Metastatic angiosarcoma with strongly positive endothelial cell marker CD 31. Magnification $\times 400$.

an angiosarcoma initially resembled a pyogenic granuloma [5]. Clinically and histologically the lesions may display many variations and early biopsy is mandatory with immunohistochemical characterization [6,7]. Misjudgment and late diagnosis may seriously affect treatment outcome. In patients with soft-tissue sarcomas, survival after 5 years ranges from $46 \%$ to $71 \%$ in the primary or metastasis condition of the disease [6,8,9].

Only 53 cases of oral angiosarcomas were reported in the literature by 1986. Jawbone lesions from this tumor are even more rare [10].

Oral angiosarcomas are also very aggressive tumors. Favia et al. reported on four cases where no patient survived more than 6 months after diagnosis [11]. These tumors were regarded as primary tumors of the oral cavity, whereas our case was a metastasis to the mandible, with a survival period of 26 months from diagnosis. This survival time can be related to the mean survival times for cardiac sarcomas reported by other authors that were in the range of 9 to 16.6 months [12]. We have found very few papers reporting a metastasis to the jaws from a primary tumor of the heart. This is in accordance with Eisenberg et al. who in 2007 reported on a case of maxillary metastasis from a primary tumor of the left atrium of the heart with metastases to the brain. This tumor was however different to the one we describe here as it was histo-pathologically described as undifferentiated highgrade cardiac sarcoma [13].

Metastasis to the oral cavity from cancers elsewhere in the body is not very common but may in some cases be the first sign of the primary tumor. In aextensive review, Hirschberg et al. collected and reviewed 673 cases between 1916 and 2006, where inclusion criteria of the primary origin of the tumor, the oral site and histological confirmation were reported. The most common metastatic sources to the oral cavity weretumors of the lung, kidney, liver and prostrate in the male patient and breast, 
female genital organs, kidney and colorectum in the female. In their material, the oral cavity represented the first location of metastatic spread in $25 \%$ of the cases and in $23 \%$ was the first indication site of an undiscovered cancer from a distant primary tumor. Jawbone is in some cancer types a common target for metastatic spread such as from prostate gland in men and breast in females [7].

Angiosarcoma is a rare tumor of vascular endothelium, and is most commonly seen in the skin, especially in the scalp of the head and neck region. Primary or metastatic diagnosis of angiosarcoma occurs only in $4 \%$ of the pharynx, oral cavity or paranasal sinuses [14]. In the literature the prognosis factor is based on size, depth and grade of the tumors and the overall prognosis is very poor [815].

Penel et al. retrospectively studied the prognostic factor in general of head and neck sarcomas in 45 adults (10 patients with histological subtype of angiosarcoma) noting that the quality of surgery (numbers of procedures and surgical margins) plays an important role for survival prognosis. Furthermore, soft-tissue sarcomas are classified as grades in a three-scale system (necrosis, differentiation and mitosis rate) and it has been shown that the 3-year survival rate decreases drastically in compareson of grade 1 - 3 where 3 represents the lowest chance of metastasis free survival [6-16].

To improve the survival rate of patients with sarcomas of the head and neck, it has been emphasized that the best treatment quality is based on an experienced multidisciplinary group approach delivering a correct and early diagnosis and thereafter-adequate resection surgery with sufficient margins [6].

The case presented in this paper displays several areas of discussion. Early clinical diagnosis may sometimes be difficult because of the rarity of the tumor but the histopathologic findings are often rather typical and can be supplemented routinely with immunohistochemistryto aid the confirmation of the histologic diagnosis. Markers like CD31, CD34, and Factor VIII-associated antigen (von Willebrand factor) for vascular endothelia are tested for and found present in the lesions. Positivity for these markers confirms the endothelial phenotype that is found malignant vascular tumors [17]. The metastasis to the mandible a year later in our case displayed the same histological pattern as the primary tumor found in the heart.

Metastases from angiosarcomas are a sign of hematogenous widespread disease as in our case where the lung, liver and finally the mandible were affected. It has been reported that one third of the metastases from angiosarcomas are found in the head and neck area [7]. Eventually our patient sustained a pathological fracture due to tumor and radiation. A previous case of angiosarcoma of the mandible causing a pathological fracture has been reported, with disseminated disease in the chest and pelvis but with normal cardiac function [18].

The clinical course for the patient in our case thereafter included a necessary stabilization of the mandible with resection and installation of a reconstruction plate. The necrotic resected bone tissue was subject to analysis for tumor growth and signs of viable tumor was found in the anterior part of the resected bone. As the tumor cells spread hematogenously, there has been interest in evaluating the bone marrow involvement in angiosarcoma, especially from the spleen as a primary source of tumor spread. A high degree of micro-metastases in the bone marrow can be seen in patients with common malignancies and the additional diagnostic tool of bone marrow aspirates for diagnosis of spread disease has been suggested [17]. In our case tumor-tissue could be found in the area even though previous radiation to the mandible.

\section{CONCLUSION}

We present a case of primary angiosarcomaof the heart with multiple metastatic regions to the lung, liver and finally the mandible. Even though other serious metastases were already confirmed; the diagnosis of mandible metastasis was postponed till histopathology results were final. However, this highlights the importance of constant awareness of possibility of metastatic spread to the oral and maxillofacial area from cancer in other areas of the body.

\section{REFERENCES}

[1] Skubitz, K.M. and D’Adamo, D.R. (2007) Sarcoma. Mayo Clinic Proceedings, 82, 1409-1432. doi:10.4065/82.11.1409

[2] Young, R.J., Brown, N.J., Reed, M.W., Hughes, D. and Woll, P.J. (2010) Angiosarcoma. The Lancet Oncology, 11, 983-991. doi:10.1016/S1470-2045(10)70023-1

[3] Lydiatt, W.M., Shaha, A.R. and Shah, J.P. (1994) Angiosarcoma of the head and neck. American Journal of Surgery, 168, 451-454. doi:10.1016/S0002-9610(05)80097-2

[4] Fanburg-Smith, J.C., Furlong, M.A. and Childers, E.L. (2003) Oral and salivary gland angiosarcoma: A clinicopathologic study of 29 cases. Modern Pathology, 16, 263-271. doi:10.1097/01.MP.0000056986.08999.FD

[5] Munoz, M., Monje, F., Alonso del Hoyo, J.R. and Martin-Granizo, R. (1998) Oral angiosarcoma misdiagnosed as a pyogenic granuloma. Journal of Oral and Maxillofacial Surgery, 56, 488-491. doi:10.1016/S0278-2391(98)90719-4

[6] Penel, N., Mallet, Y., Robin, Y.M., Fournier, C., Grosjean, J., Ceugnart, L., et al. (2008) Prognostic factors for adult sarcomas of head and neck. International Journal of Oral and Maxillofacial Surgery, 37, 428-432. doi:10.1016/j.ijom.2008.01.019

[7] Hirshberg, A., Shnaiderman-Shapiro, A., Kaplan, I. and 
Berger, R. (2008) Metastatic tumours to the oral cavityPathogenesis and analysis of 673 cases. Oral Oncology, 44, 743-752. doi:10.1016/j.oraloncology.2007.09.012

[8] Kraus, D.H., Dubner, S., Harrison, L.B., Strong, E.W., Hajdu, S.I., Kher, U., et al. (1994) Prognostic factors for recurrence and survival in head and neck soft tissue sarcomas. Cancer, 74, 697-702. doi:10.1002/1097-0142(19940715)74:2<697::AID-CNC R2820740224>3.0.CO;2-A

[9] Penel, N., Van Haverbeke, C., Lartigau, E., Vilain, M.O., Ton Van, J., Mallet, Y., et al. (2004) Head and neck soft tissue sarcomas of adult: Prognostic value of surgery in multimodal therapeutic approach. Oral Oncology, 40, 890897. doi:10.1016/j.oraloncology.2004.04.001

[10] Sengun, D., Tuncer, I., Ertem, N. and Sengun, O. (1986) Metastatic hemangioendotheliosarcoma of the mandible: Report of case. Journal of Oral and Maxillofacial Surgery, 44, 806-810. doi:10.1016/0278-2391(86)90159-X

[11] Favia, G., Lo Muzio, L., Serpico, R. and Maiorano, E. (2002) Angiosarcoma of the head and neck with intra-oral presentation: A clinico-pathological study of four cases. Oral Oncology, 38, 757-762. doi:10.1016/S1368-8375(02)00045-3

[12] Murphy, M.C., Sweeney, M.S., Putnam Jr., J.B., Walker, W.E., Frazier, O.H., Ott, D.A., et al. (1990) Surgical treatment of cardiac tumors: A 25-year experience. The Annals of Thoracic Surgery, 49, 612-618. doi:10.1016/0003-4975(90)90310-3

[13] Eisenberg, E., Natarajan, E., Yamase, H. and Hatzigiannis,
G. (2007) Undifferentiated cardiac sarcoma metastatic to the maxilla: Report of a case. Journal of Oral and Maxillofacial Surgery, 65, 148-151. doi:10.1016/j.joms.2006.08.008

[14] Carr, R.J. and Green, D.M. (1986) Oral presentation of disseminated angiosarcoma. British Journal of Oral and Maxillofacial Surgery, 24, 277-285. doi:10.1016/0266-4356(86)90094-X

[15] Weber, R.S., Benjamin, R.S., Peters, L.J., Ro, J.Y., Achon, O. and Goepfert, H. (1986) Soft tissue sarcomas of the head and neck in adolescents and adults. American Journal of Surgery, 152, 386-392. doi:10.1016/0002-9610(86)90309-0

[16] Coindre, J.M., Terrier, P., Guillou, L., Le Doussal, V., Collin, F., Ranchere, D., et al. (2001) Predictive value of grade for metastasis development in the main histologic types of adult soft tissue sarcomas: A study of 1240 patients from the French Federation of Cancer Centers Sarcoma Group. Cancer, 91, 1914-1926. doi:10.1002/1097-0142(20010515)91:10<1914::AID-CN CR1214>3.0.CO;2-3

[17] Wang, C., Rabah, R., Blackstein, M. and Riddell, R.H (2004) Bone marrow metastasis of angiosarcoma. Pathology-Research and Practice, 200, 551-555. doi:10.1016/j.prp.2004.05.003

[18] Khodayari, A. and Khojasteh, A. (2005) Mandibular pathologic fracture as a first sign of disseminated angiosarcoma: A case report and review of literatures. Oral Oncology, 41, 178-182. doi:10.1016/j.ooe.2005.05.001 